\title{
On Non-Universality of Solar-Terrestrial Connections
}

\author{
Pustinik Lev ${ }^{1}$ \\ Israel Cosmic Ray and Space Weather Center of Tel Aviv University Israel Space Agency and Golan \\ research Institute, P.O. Box 39040, Tel Aviv, 69978, ISRAEL; University ITMO, Saint Petersburg, Russia; \\ E-mail: levpust@post.yau.ac.il
}

\section{Yom Din Gregory}

Israel Cosmic Ray and Space Weather Center of Tel Aviv University Israel Space Agency and Golan research Institute, P.O. Box 39040, Tel Aviv, 69978, ISRAEL; Open University, Israel; E-mail:

gregoryyd@gmail.com

\section{Dai Uri}

Israel Cosmic Ray and Space Weather Center of Tel Aviv University Israel Space Agency and Golan research Institute, P.O. Box 39040, Tel Aviv, 69978, ISRAEL; E-mail :daiuri@gmail.com

\begin{abstract}
Discussion on principal possibility of causal chain from solar activity and space weather to the earth weather and agriculture price dynamics continue more than 200 years from first publication of Herschel (1801) up to the current time. We analyze main arguments of two sides and show that root of the critics of this possibility based on wide accepted conception in universality of solar-terrestrial connection (like to daily and season variations) what suggest that effect must be observed in any historical periods and in all regions. We show that expectation is not correct because solar-terrestrial connections generated by different sides of solar activity with different agents of solar magnetic dynamo process have different and non-stable phase pattern. We remind that realization of causal chain "solar activity/space weather" - "earth weather" - "crops" - "market reaction" may have place only in specific historical periods and in specific zone where and when the three necessary conditions will be complete. This limitation leads to possibility of four possible scenarios of market reaction. We show that the critical arguments used for rejected principal possibility of causal connection "solar activity" - Earth agriculture markets" based on ignoring of necessity of 3 necessary condition for realization of the causal chain and using for analysis periods and location, when and where necessary condition are not completed
\end{abstract}

The 34th International Cosmic Ray Conference

30 July- 6 August, 2015

The Hague, The Netherlands

\footnotetext{
${ }^{1}$ Speaker 


\section{Introduction}

Specific property of solar-terrestrial connection (STC) is "non-universality" of their manifestation. Both amplitude and phase of response may change drastically depend on historical period and location on the Earth. The non-universality is caused by complex character of solar activity - main driver of space weather influence on the Earth environment. Dynamo mechanism of generation of solar magnetic fields includes few components, interacted one with another: convection zone, differential rotation and global meridional circulation from one side, global magnetic field and small scale fields of active regions from other side. Each from this component participates in dynamo process during 22-year solar activity cycle with its specific phase trajectory shifted and distorted relative to another one. This pattern is not stable and conservative, but change drastically on time tens and hundreds years up to full suppressing of some elements of solar activity during a long time (like to Maunder minimums of sunspots and another same). Observed space weather elements are result of the modulation of condition in the nearest space and in the Earth environment by one from these solar activity drivers (or by combination of part from them). In result different space weather factors (like sunspots and solar corona, UV and X-ray emission, solar flares, coronal holes, solar wind with its sectorial structure, coronal mass ejection, solar cosmic ray and other) have specific and unstable phase portrait, not similar to another one. Since solar activity as driver is not stable process, space weather influence on the Earth must by variable and non-stable too.

One of the most discussable themes of solar-terrestrial activity is connection between solar activity and prices on agriculture production declared firstly as scientific result by famous William Hershel (1801). The situation with substantiation or disproof of this statement is much more complex because suggested scheme of possible connection includes very long multielements causal-reason chain: "solar activity”-“space weather”-“earth weather”-“agriculture production"-"market reaction on deficit". Each from these elements has different and variable sensitivity, what may change in time and depended on location of selected region on the Earth. As we show in earlier publications Pustil'nik, Yom Din (2004a, 2004b, 2013) it is possible realization of the four different scenarios of this causal chain, depended on climate zone, specific sensitivity crops to weather abnormalities, state of the market (level of globalization) and local weather sensitivity to space weather factors. In result expected price reactions on space weather abnormalities are not universal, but change its amplitude (up to negligible and, even up to change of the sign of reaction to opposite) depend on local condition.

Observed non-universality and not-stability of detected solar-terrestrial connections initiates natural skepticism of opponents with suspicions that published arguments, supported reality of this relation in specific locations and during specific historical periods, are result of manipulations with observational data, when data contradicted to authors hypothesis ejected from the sample and selected only sub-samples, what are in good agree with point of view of authors. Certainly, this suspicious absolutely correct when data filtration (choosing location and time period for analysis) has place posteriori after preliminary data analysis. In opposite case, when choosing of location and historical period has place before data analysis on the base of external criteria, formulated a priori, this accusations are not correct. 


\section{Three necessary conditions and four possible scenarios.}

We would like to remind that thesis on non-universality of causal-reason chain: "solar activity”-“space weather”-“earth weather”-“agriculture production”-“market reaction on deficit” was formulated in Pustil'nik, Yom Din (2013) in form of three necessary conditions for the implementation of the cause-and-effect relationship between the space weather anomalies and the grain price hikes they cause.

1. High sensitivity of weather (local in space and time) to the factors of space weather (e.g., cosmic rays, solar UV emission and/or magnetosphere activity).

2. High sensitivity of the leading grain harvests to the weather anomalies (belonging to the area of "high risk farming") in the given region during the studied historical period.

3. High sensitivity of the grain market to the supply deficit due to the limitation or absence of the external supply, leading to the explosive (panic) price growth.

Only simultaneous realization these three necessary conditions in studied region and in studied historical period give chance to detect manifestation of possible influence of space weather and solar activity on agriculture markets. Four possible scenarios of market reaction on solar activity are described (see Fig 1.) and it is shown that the both amplitude of market reaction and sign of the reaction depend on the type of climate dominating in the studied region.

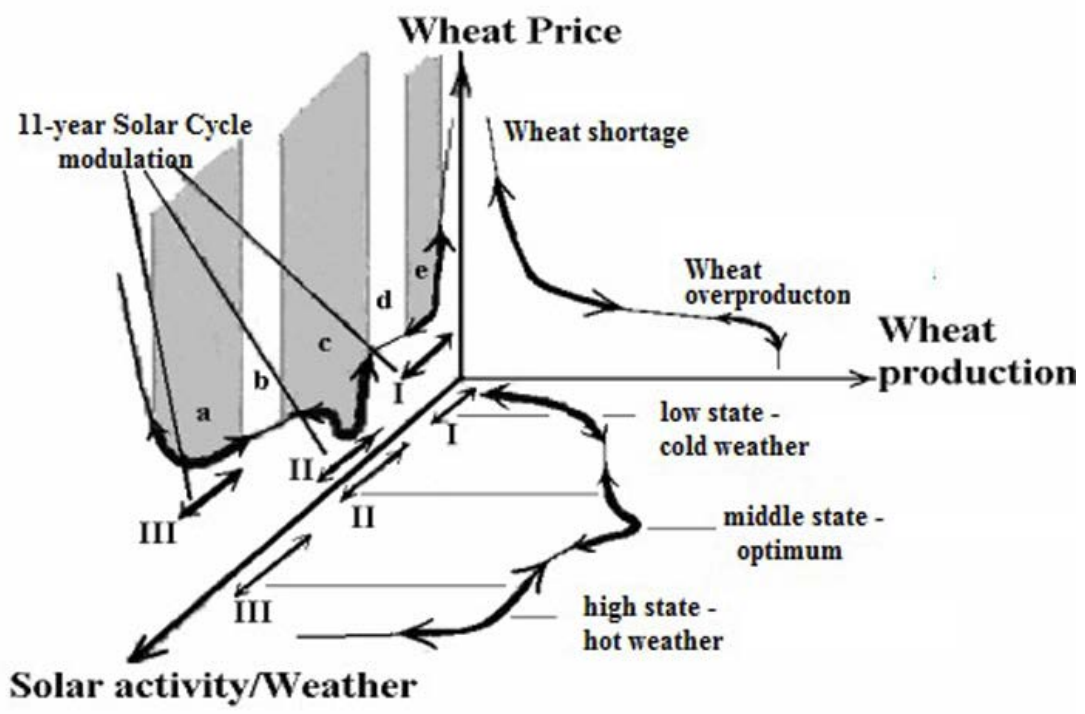

Figure 1. 3-D presentation of 4 different scenarios of possible agriculture response on space weather abnormalities through Earth weather reaction.

For example, the sign of the reaction in humid and cold zones with deficit of irradiation and heat may be opposite to the sign of the reaction in the zone with dry and hot climate with deficit of precipitation and deficient moister in soil. Violation of even one of these necessary conditions in selected region during selected historical period makes the chances of detecting of influence of solar activity on wheat market for his location small or negligible. 
Possible reasons for violating the above-mentioned cause-and-effect relationship may include:

- Choosing a region whose atmospheric conditions are of low sensitive to space weather factors: cosmic rays, UV solar emission, magnetospheric activity2

- Choosing a region where agricultural production is stable and of low sensitivity to weather abnormalities ("low risk farming”);

- Choosing agriculture markets highly open to globalization, with free trade of agricultural production between different regions and with low import costs. This globalization supports the "law of one price".

Besides the presented reasons for possible suppression of the cause-and-effect relationship the researcher can diminish these effects using samples of data taken from different regions including those with opposite types of price reaction or regions of low sensitivity to space weather. This mixing together data from heterogeneous sources (meaning different or opposite sensitivity to space weather and earth weather) can suppress observable effects and to prevent detecting of possible solar-terrestrial connection.

\begin{tabular}{|c|c|c|c|}
\hline \multicolumn{4}{|c|}{$\begin{array}{l}\text { Correlation between solar activity (sunspot number) and magnetosphere } \\
\text { activity (aa) during few historical intervals }\end{array}$} \\
\hline $\begin{array}{l}\text { Historical } \\
\text { period }\end{array}$ & $\begin{array}{c}\text { Correlation } \\
\text { coefficient for } \\
\text { historical period }\end{array}$ & $\begin{array}{c}\text { Number of } \\
\text { measurements }\end{array}$ & $\begin{array}{l}\text { Confidence interval of } \\
\text { correlation for } 95 \% \\
\text { confidence level }\end{array}$ \\
\hline 1868-2009 & 0.54 & 144 & 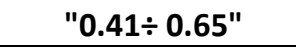 \\
\hline $1965-2009$ & 0.25 & 31 & $"-0.11 \div 0.63 "$ \\
\hline 1965-1985 & 0.01 & 21 & "-0.42 $\div 0.44 "$ \\
\hline
\end{tabular}

As illustration of non-universal character we would like to demonstrate change in correlation between solar activity in form of sunspot index and magnetospheric activity (see Fig. 2) base of many STC. According to table 1. correlation between sunspot activity and "aa" index is reliable during full period of observations (1986-2009) with 95\% confidence interval for correlation $r_{\text {min }} \div r_{\max }=0.41 \div 0.65$, during final 45 year situation was opposite: $95 \%$ confidence level for correlation coefficient $\mathrm{r}_{\min } \div \mathrm{r}_{\max }=-0.11 \div 0.6$ covered $\mathrm{r}=0$ (equivalent to absence of correlation) and for 20 years period 1965-1985 confidence 95\% interval is $r_{\min } \div r_{\max }=-0.42 \div 0.44$ (include possibility of high anti-correlation). This result does not mean that hypothesis about STC "solar activity”-“magnetospheric activity” is incorrect. In really, observed abnormalities in correlation between "aa" and "SN" lead us to rejection only of hidden assumption about universal, stationary and stable character of this relation. It demonstrate, that dependence of geomagnetic activity from the solar one is non-stationary and non-linear, sensitive to phase portrait of manifestations of different elements of solar dynamo process (see for articles of Georgieva-Kirov

\footnotetext{
${ }^{2}$ We remind here that according last year investigations this sensitivity has very inhomogeneous distribution in space and is unstable in time (see for example map of sensitivity cloudiness to cosmic ray variation (Marsh and Svensmark, 2000). As another example of complex character of possible influence of geomagnetic activity on North Atlantic Oscillation factor, controlled weather in North hemisphere see article of Li et al.,(2011).
} 
(2011) and Krischa et all (1999). Similar non-universal situation is standard for solar-terrestrial connections and must be base for analyzing of observed relations.

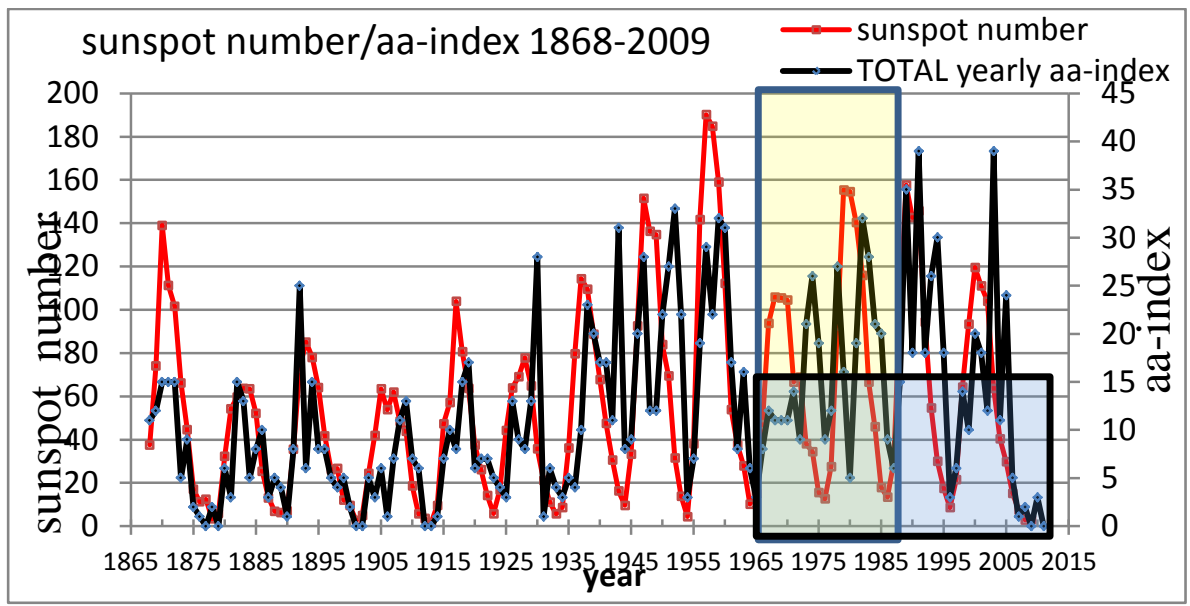

Figure 2. Variation of sunspots numbers and geomagnetic activity index "aa” during 1986-2009 years. Yellow and blue regions cover interval of insignificance correlations (1965-2009 and 1965-1985 periods, correspondingly)

\section{Acknowledgements}

This research was supported by grant ISA of Ministry of Science of Israel in the frame of the "Center of Knowledge in Cosmic Ray Research".

\section{References}

[1] Georgieva, K., Kirov, B., "Solar Dynamo and Geomagnetic Activity", Journal of Atmospheric and Solar-Terrestrial Physics, vol. 73, Iss. 2-3, 207-222, 2011.

[2] Herschel, W., "Observations tending to investigate the nature of the Sun, in order to find the causes or symptoms of its variable emission of light and heat; With remarks on the use that may possibly be drawn from solar observations”, Phil. Transactions of. Royal Society of London, vol. 91, 265-318, 1801.

[3] Li Yu., Lu H., Jarvis M.J., Clilverd M.A., and Bates B., "Nonlinear and nonstationary influences of geomagnetic activity on the winter North Atlantic Oscillation", Journal of Geophysical Research, vol. 116, 3-15 , doi:10.1029/2011JD015822, 2011.

[4] Love J.J., "On the insignificance of Herschel's sunspot correlation”, Geophysical Research Letters, vol. 40, Issue 16, pages 4171-4176, 2013.

[5] Marsh, N. D. and Svensmark, H., "Low Cloud Properties Influenced by Cosmic Rays”, Phys. Rev. Lett., vol. 85, 23, 5004-5016, 2000.

[6] Pustil'nik L., Yom Din G., "Influence of solar activity on the state of the wheat market in medieval England”, Solar Physics, 223, 1, 335-356, 2004a.

[7] Pustil'nik L., Yom Din G., “Space Climate Manifestation in Earth Prices-from Medieval England up to Modern USA”, Solar Physics, 224, 1-2, 473-481, 2004b.

[8] Pustil'nik L., Yom Din G., “On possible influence of space weather on agricultural markets: Necessary conditions and probable scenarios", Astrophysical Bulletin (Pleiades Publishing), vol. 68, 1, pp.107-124, 2013. 